\title{
Dietary restriction misconceptions and food allergy education in children with atopic dermatitis
}

You Hoon Jeon, MD, PhD

Department of Pediatrics, Hallym University Dongtan Sacred Heart Hospital, Hwaseong, Korea

\section{Key message}

- Food intake strategies for preventing food allergies have undergone major changes over the past 20 years.

- In children with atopic dermatitis, indiscriminate food restrictions without diagnostic testing leads to nutritional imbalance and poor growth.

- When determining food restrictions for pediatric patients with atopic dermatitis, an accurate food allergy diagnosis must be preceded, and continuous parental education about food intake is required.

Education for caregivers and patients regarding allergic diseases should be emphasized. In particular, atopic dermatitis is the starting point of allergic march in food allergies, allergic rhinitis, and asthma, and it renders the continuous education of parents important to ensuring their satisfactory understanding and management of atopic dermatitis, specifically with respect to the preventive treatment of allergic diseases. Parents of children with atopic dermatitis should be thoroughly educated on skin care routines, such as bathing and moisturizing, as well as environmental management and the consumption or avoidance of foods that can be trigger factors. In most guidelines for managing atopic dermatitis, correct skin care, patient education, and the avoidance of identified allergens constitute basic therapy at all severity levels. ${ }^{1)}$ The problem is that the avoidance of causative antigens, particularly food allergens, is often insufficiently understood by caregivers, in part because of major changes in the paradigm of food allergy prevention (Fig. 1).

In 2000, the American Academy of Pediatrics recommended

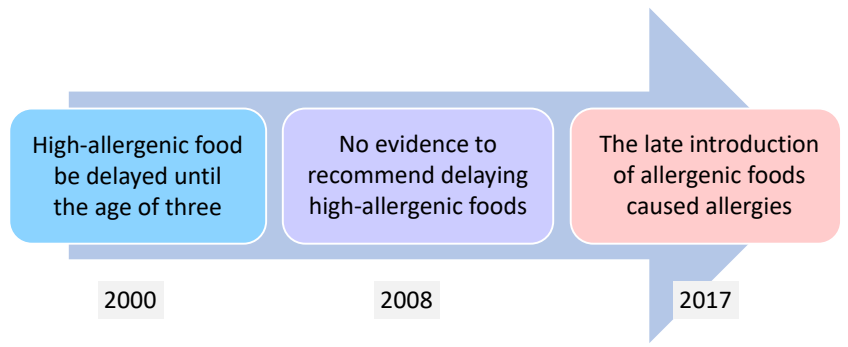

Fig. 1. Paradigm changes in food allergy prevention. that the introduction of highly allergic food be delayed (dairy products until 1 year of age, eggs until 2 years of age, and peanuts, nuts, and fish until 3 years of age) in infants with a considerable risk of allergies. ${ }^{2)}$ These guidelines, however, have rapidly changed over the next 20 years. In 2008, they mentioned that there is no need to postpone the introduction of solid foods beyond 4-6 months of age as a means of preventing food allergies. ${ }^{3)}$ In 2015, the Learning Early About Peanut Allergy (LEAP) study reported that the concept of food consumption to prevent food allergies has completely changed. This study targeted 640 high-risk infants aged 4-11 months with severe eczema, egg allergy, or both. Peanut allergies occurred much more frequently in the peanut avoidance group than in the peanut consumption group. ${ }^{4}$ ) The LEAP-On study results were similar to those of the LEAP study. Therefore, studies of the early introduction of other foods, such as eggs and milk, have been conducted. These studies suggested the possibility that early introduction will prevent food allergies. ${ }^{5-7)}$ The strategy of introducing allergic food as late as possible is no longer recommended, as it is thought to negatively affect the prevalence of food allergies. An exception occurs when a child already shows allergic reactions to certain foods. For these foods, the priority principle involves refraining from consuming them until a patient passes the food challenge test or allergy test results improve and symptoms disappear.

In a study by Youm et al., ${ }^{8)} 72.8 \%$ of children with atopic dermatitis were started on solid food after 6 months of age. This approach was presumed to have been driven by the vague anxiety of parents that "specific foods will worsen my child's atopic dermatitis" or that "food allergies will always accompany atopic dermatitis." The reality is that only one-third of moderate to severe pediatric atopic dermatitis cases are accompanied by food allergies. Moreover, recent studies have shown that late weaning can contribute to the occurrence of food allergies and that problems related to eating habits and development can arise. ${ }^{9,10)}$ Without a food allergen sensitization evaluation and an accurate food allergy diagnosis, the restriction of certain foods simply because they generally cause allergies at high frequencies can lead to nutritional imbalances and negative effects on a child's growth. In Malaysia, a study was conducted on children 
aged 12-36 months with atopic dermatitis. The diets of $60.7 \%$ of the cases were restricted to three or more foods, with these children having lower intakes of calories, protein, carbohydrate, fat, riboflavin, vitamin B12, phosphorus, calcium, and iron. The $\mathrm{z}$ scores for weight, height, head circumference, midupper arm circumference, and body mass index of the children with dietary restrictions were significantly lower than those without such interventions. ${ }^{11)}$ Youm et al. ${ }^{8)}$ reported that $39.7 \%$ of the instances of food restriction for children with atopic dermatitis were determined by parental judgment; of these cases, $44.4 \%$ were decided without the presence of symptoms or a medical evaluation. This phenomenon indicates that parents incorrectly understand atopic dermatitis and food allergies due to a lack of education on the latter. Parental misunderstanding that foods that frequently cause allergies in children (e.g., milk and eggs) cause food allergies in all children should be corrected. Food allergies should be diagnosed based on laboratory results, and a detailed history or oral food challenge tests and regular tests should be performed to determine whether children can outgrow their food allergies and consume a particular food.

Given that children must continue to grow and develop, the diagnosis of food allergies and decisions regarding diet restrictions in this population should be made carefully. Parents also require proper education with these matters.

See the letter "Environmental and dietary factors to be checked for treatment of atopic dermatitis in rural children" via https://doi.org/10.3345/cep.2021.00605.

\section{Footnotes}

Conflicts of interest: No potential conflict of interest relevant to this article was reported.

ORCID:

You Hoon Jeon (1) https://orcid.org/0000-0002-8164-7580

\section{References}

1. Wollenberg A, Christen-Zäch S, Taieb A, Paul C, Thyssen JP, de BruinWeller M, et al. ETFAD/EADV Eczema task force 2020 position paper on diagnosis and treatment of atopic dermatitis in adults and children. J Eur Acad Dermatol Venereol 2020;34:2717-44.

2. American Academy of Pediatrics. Committee on Nutrition. Hypoallergenic infant formulas. Pediatrics 2000;106:346-9.

3. Greer F, Sicherer S, Burks A. Effects of early nutritional interventions on the development of atopic disease in infants and children: the role of maternal dietary restriction, breastfeeding, timing of introduction of complementary foods, and hydrolyzed formulas. Pediatrics 2008;121: 183-91.

4. Du Toit G, Roberts G, Sayre PH, Bahnson HT, Radulovic S, Santos AF, et al. Randomized trial of peanut consumption in infants at risk for peanut allergy. NEngl J Med 2015;372:803-13.

5. Du Toit G, Sayre PH, Roberts G, Sever ML, Lawson K, Bahnson HT, et al. Effect of avoidance on peanut allergy after early peanut consumption. $\mathrm{N}$ Engl J Med 2016;374:1435-43.

6. Bird JA, Parrish C, Patel K, Shih JA, Vickery BP. Prevention of food allergy: beyond peanut. J Allergy Clin Immunol 2019;143:545-7.

7. Abrams EM, Sicherer SH. Cow's milk allergy prevention. Ann Allergy Asthma Immunol 2021;127:36-41.

8. Youm S, Lee E, Lee J. Environmental and dietary factors to be checked for treatment of atopic dermatitis in rural children. Clin Exp Pediatr 2021; 64:661-31.

9. West C. Introduction of complementary foods to infants. Ann Nutr Metab 2017;70:47-54.

10. Przyrembel H. Timing of introduction of complementary food: shortand long-term health consequences. Ann Nutr Metab 2012;60:8-20.

11. Low DW, Jamil A, Md Nor N, Kader Ibrahim SB, Poh BK. Food restriction, nutrition status, and growth in toddlers with atopic dermatitis. Pediatr Dermatol 2020;37:69-77.

How to cite this article: Jeon YH. Dietary restriction misconceptions and food allergy education in children with atopic dermatitis. Clin Exp Pediatr 2022;65:83-4. https://doi. org/10.3345/cep.2021.01634 